\title{
Weitere Untersuchungen über das Riechepithel und sein Verhalten zum Nervus olfactorius.
}

$$
\text { Von }
$$

\author{
Dr. A. v. Brumn,
}

Prosector in Göttingen.

Hierzu Tafel XI.

Die der folgenden Mittheilung zu Grunde liegenden Untersuchungen wurden in der Absicht angestellt, der Beantwortung der Frage nach der Endigung des Riechnerven nïher zu kommen, als das bisher geschehen ist. Die letzte Endigung dieses Nerven habe ich nicht gefunden, iiberhaupt mutss ich leider gestehen, dass grade in Bezug auf diesen Hauptpunkt wenig Neues zu Tage gefördert wurde. Dennoch scheinen mir die Resultate der Mittheilung werth. Sie sind geeignet, die von Exner in drei Mittheilungen (Sitzungberichte d. kaiserl. Akademie der Wissenschaften zu Wien 1870, 1872, 1877) vertretene Ansicht, dass die Aeste des N. olfactorius in ein weitmaschiges protoplasmatisches subepithelial gelegenes Netzwerk übergehen, aus dem die beiden Zellenarten des Epithels - M. Schultze's Epithelial- und Riechzellen - hervorgehen, zu beseitigen; sie bestätigen und erweitern die von Babuch in (Strickers Handbuch der Lehre von den Geweben, II. Band, Leipzig 1872) vorgetragene Lehre, dass die Nervenäste direkt in das Epithel eintreten.

Selbstverständlich musste ich meine Aufmerksamkeit auch wiederholt den Elementen des Epithels zuwenden und aufs Neue die von mir (Archiv für mikr. Anatomie Band XI) beschriebene Membrana limitans olfactoria, welche von Exner in seinem dritten Aufsatze und von Löwe (Beiträge zur Anatomie der Nasenund Mundhöhle, Berlin 1878) geleugnet wird, auf ibre Existenz 
untersuchen, sowie auch die das Epithel gegen die Schleimhaut abgrenzenden Theile ins Gebiet meiner Untersuchungen ziehen.

Zunächst ein paar Worte üher das Epithel. Ich muss hier die M. Schultze'sche strenge Scheidung von Epithelial- und Riechzellen auch jetzt noch durchaus festhalten, wiewohl ich Exner das wenn auch recht seltene Vorkommen von Uebergangsformen zugestehe. Ich muss diese Scheidung ftir durchaus nothwendig halten, weil das Verhaiten der Zellenarten zur Oberfläche ein durchaus verschiedenes ist, wie ich spüter darlegen werde. - Dagegren bestätige ich die von W. Krause (C. F. Th. Krause's Handbuch der menschlichen Anatomie, III. Anfl., Band I. Hannover 1876) als Basalzellen beschriebenen und von Mahmoud Sidky (Recherches anatomo-microscopiques sur la muqueuse olfactive, Thèse de Paris 1877) als Couche basilaire zusammengefassten Zellen und kann als besonders guinstiges Object zu ihrer Darstellung die Riechschleimhaut der Vögel (Huhn, Ente, Taube) empfehlen, wo diese Gebilde eine sehr regelmïssig conische Gestalt besitzen und besonders lang und zahlreich sind, was hier namentlich gegeniiber der geringen Zahl der Riechzellen auffaillt.

Was nun das Verhalten der Epithelial- und Riechzellen zur Oberfliche betrifft, so habe ich in dem oben citirten Aufsatze eine diese Fläche bedeckende, wie ich damals meinte, amorphe Substanzschichte beschrieben und abgebildet, die eine nicht unbetrïchtliche Dicke, etwa $0,005 \mathrm{~mm}$ hat und auf ibrer unteren Fläche Leisten besitzt, welche zwischen die aneinander stossenden Epithelialzellen eine kurze Strecke eindringen, und durch welche diese Schicht, wenn man sie isolirt von der Fläche sieht, eine netzförmige Zeichnung erkennen lässt. In den Leisten befinden sich Poren, welche die ganze Schicht durchsetzen, und in denen die peripherischen Fortsätze der Riechzellen stecken, so dass sie also direkt von der die Nase durchstreichenden Luft getroffen werden, von der die Epithelialzellen durch eben diese Schicht getrennt sind; ich nannte diese Schicht Membrana limitans olfactoria. Seitdem hat W. Krause die Entdeckung gemacht, dass das Riechepithel von änsserst feinen erst bei 800 maliger Vergrösserung erkennbaren, nicht flimmernden Härchen bedeckt ist, er schreibt solche den beiden Zellenarten zu, meint aber, sie scheinen auf den Riechzellen etwas dicker zu sein. Derselbe erkennt zugleich aber die Membr. limitans an; ebenso Sidky, - nur dass Letzterer 
meine Angabe dahin modificirt, dass die genannte Begrenzungshaut unmessbar dünn sei; die Krause'schen Härchen kennt er nicht. Löwe bestätigt die Letzteren. Er erklärt das Verhältniss der Epithelial- und Riechzellen zur Oberfläche für gleich, lässt beide mit denselben Härchen besetzt sein, erkennt als Besonderheit des Flimmerbesatzes der Regio olfactoria nur seine leichte Abhebbarkeit in grossen Stücken an, und meint, meine Limitans sei identisch mit dem Härchenbesatz beider Zellenarten. Exner in seiner dritten Mittheilung ist derselben Ansicht bezüglich des Frosches, wïhrend er die Härchen bei Säugethieren noch nicht allgemein anerkennt.

Auch diese Krause'sche Entdeckung kann ich, wenn auch mit einiger Beschränkung, bestätigen. Es findet sich über den Epithelialzellen ein Satm, der bei Hartnack 10 à imm. dentliche Streifung senkrecht zur Oberfläche zeigt, dessen vollständige Auflösung in einzelne Härchen aber kaum vollkommen gelingt. Es verhält sich mit ihm ungeführ, wie mit dem Saume der Darmepithelzellen, an dem man ja auch gelegentlich eine Isolation der ihn zusammensetzenden Stäbchen beobachtet. Indessen stehen, glaube ich, die Stäbchen, aus denen sich jener Saum zusammensetzt, den Flimmerhaaren noch näher, als die Elemente des Epithelialzellenbesatzes der Riechschleimhaut. - Trotzdem ist die Limitans nicht aufzugeben: sie liegt vielmehr unter den Härchen - rudimentäre Flimmerhaare könnte man sie vielleicht nennen, - sie bedeckt die Epithelialzellen, besitzt entsprechend den Grenzen dieser die Verdickungen, die ihr in der Flächenansicht das netzförmige Ansehen geben und auf dem Durchschnitt an der unteren Fläche deutlich hervortreten; sie hat von eben solchen Verdickungen umgebene Poren, durch welche die peripherischen Riechzellenfortsätze dringen, um ungefähr im Niveau der freien Enden der Härchen $z u$ endigen. Meine frühere Limitans besteht also aus dieser und den Härchen.

Dies Verhalten ergiebt sich aus Bildern, welche man auf verschiedene Weise erhalten kann. Einmal findet man in Zerzupfungspräparaten von Riechschleimhäuten, welche 24 Stunden lang in Osmiumsäure von $2 \%$ gelegen hatten und dann in Wasser macerirt waren (Fig. 1) direct, dass die Riechzellenenden durch die Poren dringen; ganz ähnliche Bilder geben Durchschnitte von Schleimhäuten, welche in Mïller'scher Flüssigkeit oder 6 proc. Lö- 
sung von Kali bichrom. und dann in Alcohol gehärtet waren, sowie auch Zerzupfungspräparate aus den genannten Flüssigkeiten. Dann mache ich darauf aufmerksam, dass man an Flichenansichten des Epithels, bei denen die freie Fläche nach Oben liegt, beim Heben des Tubus die optischen Durchschnitte der Riechzellen noch deutlich erkennt, wenn die Contouren der Epithelialzellen bereits verschwunden sind. Ich betone besonders, dass ich 2procentige Osmiumsäure anwendete, welche eine Quellung der Epithelialzellen fast gar nicht bewirkt und der man wohl auch cine Quellung der Riechzellen kaum zusehreiben kann.

Die freien Enden der Riechzellen nun finde ich an Präparaten, welche 24 Stunden in $1-2$ proc. Osmiumsäure gelegen und danach 4-6 Wochen in der Merkel'schen Mischung von gleichen Theilen Glycerin, Alcohol und Wasser zugebracht hatten, - weniger gut nach Wassermaceration - kolbig angeschwollen und mit Hïrchen von grosser Feinheit, die aber viel deutlicher sind, als jene der Epithelialzellen, spürlich besetzt. Am besten gelingt aber ilre Darstellung an Riechschleimbäuten von Säugethieren, die in der von Pacin i angegebenen Conservirungsflitssigkeit - es ist die erste der bei den Pacini'schen Mischungen, welche Frey auf S. 136 der 6. Anflage des Mikroskop angiebt und in welcher rothe Saingetbierblutkörper sich so gut halten - einige Stunden gelegen haben. Hier sind die Endflichen der Epithelialzellen sïmmtlich durchaus glatt, jede Spur der Härchen ist verschwunden, - ebenso constant aber iiberragen die Riechzellen die Oberflaiche und zeigen auf das Schönste die Riechbärchen" (Fig. 2). Auch isoliren sich lie einzelnen Zellen häufig, wenigstens der peripherische Fortsatz mit dem Zellkörper, und beseitigen so jeden Zweifel darüber, dass auch wirklich nur die Riechzellen die Träger dieser eigenthïmlichen Bildung seien. Die kurzen Härchen stehen nach allen Seiten hin von dem kolbigen Ende $a b$ und es bilden die aussersten etwa einen Winkel von $180^{\circ}$ mit einander. - Die regelmässige Erhaltung der Riechhaare und ebenso' vollständige Lösung des Härchenbesatzes der Epithelialzellen ist zugleich ein, wie ich denke, sicherer Beweis fürr die Ungleichwerthigkeit beider.

Bei Vögeln - Huhn, Ente, Taube - und Amphibien, - Frosch, Salamandra macnlosa, Triton taeniatus und alpestris - sind die Verhältnisse in mehreren Punkten andere, als bei Säugethieren. Einmal fehlen, wie ich heute noch behaupten muss, den Epithe- 
lialzellen die Haare, - ohne allen Zweifel tragen sie nicht die gleichen, wie die Riechzellen, - zweitens ragen die peripherischen Riechzellenfortsätze nicht so weit über die Oberfläche empor, sondern wölben sich nur eben halbkugelig über dieselbe, drittens sind die Riechhaare sehr viel lünger. Das Erste halte ich für eine wesentliche Differenz, die beiden anderen Punkte für unwesentliche, da sonst das Verhalten zur Limitans ganz das gleiche ist. Diese habe ich nämlich jetzt, was mir früher nicht gelungen war, anch bei allen diesen Thieren in grossen Stücken isolirt erhalten. Der Durchschnitt - am besten an geeigneten Stiicken von Zupfpräparaten aus Osmiumsäure von 2 pCt. - zeigt die glatte Limitans und durch deren Lücken die Riechzellen hindurehtretend, welche auf ihrem Endlsnöpfchen den Büschel langer Haare zeigen. Diese letzteren strahlen pinselförmig aus, sodass die äussersten einen Winkel von $30-40^{\circ}$ mit einander bilden und die von benachbarten Zellen einander vielfach durchkreuzen, wodurch das Bild eines vollständigen Wimperkleides dickerer Stücke hervorgerufen wird. Dem entspricht ausserordentlich schön die Flächenansicht. Bei hoher Tubnsstellung sieht man die punktförmigen optischen Querschnitte der Härchen ganz gleichmässig vertheilt. Senkt man den Tubus, so treten diese Pïktchen zu Gruppen zusammen, um bei weitergehender Tiefstellung mehr und mehr zusammenzuriicken und endlich auf den optischen Querschnitten der Riechzellenenden zu verschwinden. Erst bei einer abermaligen Drebung der Schraube treten dann die Leisten der Limitans auf.

So glaube ich also das gleiche Verhalten der Riechzellen zur Oberfläche des Epithels und die gleiche Beschaffenheit ihrer freien Enden bei Säugethieren, Vögeln und Amphibien nachgewiesen za haben.

Das ungleiche Verhalten der Epithelialzellen ist freilich recht unbeqnem. Die mangelhafte Ausbildung und grosse Vergänglichkeit der Kraus e'schen Härchen bei den Säugethieren lässt hoffen, dass man etwas Aehnliches bei Vögeln und Amphibien noch finden werde, es hat mir einigemale geschienen, als befinde sich iiber den entsprechenden Zellen des Frosches und Salamanders ein ähnlicher, aber viel niedrigerer Saum; indessen waren mir die Bilder zu unbestimmt, als dass ich sie für mehr als der blossen Erwähnung werth hielte.

Die gegebene Schilderng weist die von Exner und Loewe 
aufgestellte Ansicht zurtick, dass das, was ich als eine Membran beschrieben, nichts Wesentliches sei, dass das Besondere des Riechepithels nur in der leichten Ablösung des Härchenbesatzes, in grossen Stticken liege, eine Meinung, deren Unhaltbarkeit meiner Ansicht nach schon daraus hervorgeht, dass man so hänfig siehe meine frlihere Mittheilung S. 475, Fig. 7 - Präparate erhält, in denen sich die Limitans sammt dem Härchenbesatz (beide habe ich damals nicht getrennt, sondern zusammen als limitans beschrieben und bezeichnet) in grossen Stïcken isolirt findet, welche mit den festsitzenden peripherischen Riechzellenfortsätzen dicht besetzt sind, während die Epithelialzellen alle oder fast alle fehlen. Wäre das Verhältniss zur Oberfläche das gleiche, so sollte man doch bei der grösseren Oberfläche der Epithelialzellen eher an ein Festhaften dieser denken.

Als was soll man nun diese Membran ansehen? Ich stimme mit Loewe darin äberein, dass sie als Cuticularbildung aufzufassen sei: sie ist gewiss gleichbedeutend mit dem glänzenden, die Cilien tragenden Saum der gewöhnlichen Flimmerzellen, aber mit der Besonderheit, dass diese Bildung nur den Epithelialzellen zukommt und dass die den einzelnen Zellen angehörenden Säume viel fester mit einander verklebt sind, als irgendwo sonst. Wie schon in meiner früheren Mittheilung angegeben, habe ich Stücke von $0,6 \mathrm{qmm}$ isolirt gesehen. Dieser Cuticularmembran besondere Beachtung za widmen und ihr einen Namen zu geben, dazu hat man gewiss ebensoviel Recht, wie dazu, die Membr. limit. externa der Retina oder die Membr. reticularis cochleae als solche $z u$ bezeichnen, wiewohl beide von Niemandem für selbständige Bildungen gehalten werden.

Zur Verfolgung der Olfactoriusfasern habe ich mich des Goldchlorids bedient, und zwar habe ich dasselbe nach der von $L$ oewit angegebenen Methode angewandt, mit der Modification, dass ich, um die Ablösung des Epithels za verhindern, die Ameisensäure in weit schwächerer Concentration, $1-4 \%$, benutzte. Die Launenhaftigkeit des Goldehlorids bewährte sich leider auch hier. Unter etwa 50 Vergoldungsversuchen glückte nur einer recht gat: diesem einen Präparate sind die Schnitte entnommen, von denen Theile in Fig. 3 und 4 dargestellt sind. Es war dies Geruchsorgan eines fast erwachsenen Kaninchens frisch in Ameisensäure von 4\% abgespült worden, hatte dann eine halbe Stunde in Goldchlorid 
von $1 \%$ und nachher 24 Standen in derselben Ameisensäure zugebracht. Sonst habe ich in noch acht Fällen bei der Katze, dem Fuchs, dem Kaninchen, dem Huhn, Froseh und Salamander, Bilder erhalten, in denen man das Eintreten der Nerven in das Epithel deutlich erkannte, aber wegen zu tiefer Goldtinction des Epithels und der unterliegenden Schichten weniger gut. An Versuchen, eine sichere Methode ausfindig zu machen, babe ich es nicht fehlen lassen, ich habe die Concentration der Goldlösung sowohl, wie der Ameisensäure mannichfach variirt, -erstere von 0,25 bis $2 \%$, letztere von 1-20\%, - ohne zum Ziele za gelangen. Dennoch sind die einen gelungenen und die anderen leidlich gerathenen Vergoldungen durchaus beweisend dafur, dass die Aeste des Riechnerven, nachdem sie sich im Schleimhautgewebe vielfach getheilt haben und dicht unter dem Epithel noch einmal in feine Zweige zerfallen sind, direct in das Epithel eintreten; die Präparate sind durchaus nicht anders zu deuten: Fig. 3 ist ein rein senkrechter Schnitt und die Verfolgung der Nerven in das Epithel leicht. Auffallend erscheint, dass die meisten Aeste dort plötzlich, ohne zugespitzt zu sein, endigen. Flüchenschnitte erklären das leicht: sie zeigen, dass die Nerven zwischen den Füssen der Epithelialzellen plötzlich in die horizontale Richtung umbiegen und sich spitzwinklig verästeln, bis sie als feinste zugespitzte Fibrillen verschwinden. Von einer Plexusbildung im Epithel von Seiten der Nerven kann man kaum sprechen, ich wenigstens habe nie mit Sicherheit Anastomosen der Zweige beobachtet, höchstens Kreuzungen. Dass diese Verästelungen nicht subepithelial, sondern wirklich intraepithelial sind, beweisen die Flächenschnitte sowohl wie die senkrechten Durchschnitte. In ersteren hat man mit den feinen Aestchen stets zugleich die Fiisse der Epithelialzellen im Fokus; in letzteren findet man die punktförmigen Querschnitte der Fibrillen niemals unter dem Epithel, was doch bei subepithelialer Lage nothwendig der Fall sein müsste, was ganz besonders in diesen Goldpräparaten auffallen müsste, bei welchen unter dem Epithel eine völlig ungefärbte Schicht, auf, welche ich nachher noch zurückkommen werde, liegt. Innerhalb des Epithels, in den tiefsten Schichten desselben, findet man solche Ptinktchen, kann einige auch sicher als Faserquerschnitte erkennen; freilich aber ist es, da das Epithel nicht ganz farblos bleibt, in manchen Fällen schwer, wohl anch unmöglich, die Diagnose mit Sicherheit zu stellen. 
Weiter als die Abbildungen zeigen, babe ich die Verfolgung der Nerven nicht ausführen können. Vielleicht sind die Pünktchen, welche man an den feinsten Fibrillen sieht und welche denselben ein varicöses Ansehen verleihen, die optischen Durchschnitte von Aestchen, welche senkrecht im Epithel aufsteigen, es liegt dieser Gedanke nahe, da man sonst die Verjüngung der Fasern nicht recht begreift, aber direct dies Verhalten zu beobachten, habe ich nicht vermocht. B abuchin beschreibt ein Präparat von der Schildkröte, in dem ihm die Verfolgung der Nervenfasern bis an den Kern einer Riechzelle geglückt ist, - ich babe so weit nicht kommen können. Eines muss ich noch erwähnen, was ebenfalls Babuchin auffiel, dass nämlich die Körper und Fortsätze, namentlich die peripherischen, der Riechzellen - nicht aber ihre, Kerne - eine viel tiefere Goldfärbung annehmen, als die Epithelialzellen. Ich besitze Präparate, welche dies Verhalten ganz prachtvoll erkennen lassen, will aber daraus keineswegs irgend welche weiteren Schlitsse ziehen.

Meinen Abbildungen stehen die von Exner (Sitzungsberichte 1872, namentlich Taf. II, Fig. 4) gegeniiber. Er bildet dort, ebenfalls vom Kaninchen, besonders deutlich sein subepitheliales nervöses Netzwerk $a b$, in welches auf der einen Seite die Olfactoriusäste auf der anderen die Epithelial- und Riechzellen direct übergehen. Ich will eine Deutung dieses Netzwerkes nicht versuchen und überlasse es getrost der Zeit und den Nachuntersuchern, welche der beiden Abbildungen sie als die den Thatsachen mebr entsprechende erhalten, welche sie verwerfen wollen.

Endlich forderten Exner's Abbildungen zu einer genauen Durchforschung der unter dem Epithel liegenden Gewebe und zur Aufsuchung des subepithelialen Netzes auf. An den Goldpräparaten findet man unmittelbar unter dem Riechepithel, welches eine mässige Goldfärbung zeigt, eine von demselben durch geringen Glanz und absolute Farblosigkeit sich scharf unterscheidende nur ganz schwach streifige Schicht mit einzelnen, der Oberfläche pa= rallel stehenden platten Kernen, sie hat etwa 0,008 mm Dicke. Das tiefer unten die Zwischenräume zwischen den Drüsen and Nerven einnehmende Bindegewebe ist ebenfalls ziemlich scharf gegen diese Schicht abgesetzt durch bedeutend stärkere Faserung und tiefere Goldtinction. An Schnitten von in Osmiumsäure von 2 pCt. und Alcohol gehärteten Riechschleimhäuten zeigt sie-sich 
umgekehrt in einem dunkleren Farbenton, als das tiefere Gewebe. Ihre Constituentien sind an solchen Schnitten nicht zu erkennen, wohl aber kann man sich dieselben durch Zerzupfen von Präparaten, welche 14 Tage oder länger in Mtiller'scher Flüssigkeit oder Lösung von doppeltchromsaurem Kali von $2-6 \mathrm{pCt}$. gelegen hatten, zur Anschauung bringen. Man findet hier zunächst unter dem Epithel eine einfache Schicht platter Zellen, deren der Oberfläche parallel gerichtete grosse und platte Kerne vor einer Verwechselung mit den Krause'schen Basalzellen schützen (Fig. 5). Sehr oft erkennt man dies Verhältniss mit Leichtigkeit, bald an dickeren Stiicken, wo die ganze Lage der Zellen deutlich wird, bald an kleineren, ' wo nur eine solche Zelle mit den auf ihr stehenden Epithelialzellen erhalten ist; - bald anch, freilich weniger scharf, an gefärbten Schnitten durch das in 6 procentiger Lösung von Kali bichr. und dann in Alcohol gehärtete Organ. Die scharfe Abgrenzung dieser Zellen gegen das Epithel ist zweifellos.

Bringt man ein Stiick, an dem diese Zellen streckenweis ganz isolirt sind, wie das in Fig. 5 gezeichnete, zur Drehung, dann zeigt sich, dass diese Zellen sternförmige, durch zahlreiche Ausläufer mit einander anastomosirende sind (Fig. 7), zwischen denen nur hie und da eine kreisförmige Oeffnung ftir den Ausfïhrungsgang einer Bowman'schen Driise bleibt. Diese dem embryonalen reticulären Bindegewebe ähnliche Schicht bleibt nicht immer an dem Epithel, sondern häufig anch auf der nächsten Gewebsschicht haften, woraus sich wohl erklärt, dass man in manchen Zerzupfungspräparaten vergeblich nach ihr sucht. Nie vermisst habe ich sie an Flächensehnitten in Osmiumsäure gehärteter Riechschleimbäute von Säugethieren, in welchen die Zellen und Anastomosen nur etwas schlanker erscheinen, als in den Zupfpräparaten aus Chromsalzlösungen.

Diese Schicht sitzt nun einer Lage eines nur sehr undeutlich faserigen Gewebes auf, welche ungefähr dieselbe Dicke, wie die Zellen, ungefähr 0,004 mm hat, und in welcher hie und da Kerne, von etwas körniger Substanz umgeben, gefunden werden; ich bin indessen darüber nicht ins Klare gekommen, ob diese Kerne nicht etwa zusammen mit ihrer körnigen Umgebung, die auf dieser Schicht haften gebliebenen sternförmigen Zellen sind. Diese Lage homogenen Bindegewebes scheint mit den tieferen Schichten auch nur lose verbunden zu sein, wenigstens erhält man an Zupfpräpa- 
150 A. v. Brunn: Weitere Untersuchungen über das Riechepithel etc.

raten von Schleimhäuten, deren Epithel vorher vorsichtig entfernt worden war, oft grosse Sticke von ihr, deren optischer Durchschnitt - an Falten - auf beiden Seiten glatt begrenzt erscheint.

Die Deutung dieser beiden unter dem Epithel liegenden Schichten ist wohl nicht ganz leicht. Am nächsten liegt es mir, die sternförmigen Zellen den kïrzlich von Afanasieff (dieses Archiv Band XV) näher studirten ähnlich gestalteten Gebilden auf der Innenflïche der Membranae propriae der Drüsen, namentlich des Hodens, gleichzusetzen, wogegen die folgende Schicht etwa der, mitunter ja auch eine beträchtliche Dicke erreichenden Membrana propria selbst, zu vergleichen wäre.

\section{Erklärung der Abbildungen auf Tafel XI.}

Fig. 1. Aus einem Zupfpräparat der Riechschleimhaut vom Kaninchen; Osmium 1 pCt. 48 Stunden, Wasser 8 Tage. Die Epithelialzellen bedeckt von der M. limitans, ml, welche den bärchenäbnlichen Besatz trägt; aber rechts auch isolirt liegt. Die Riechzellen durchbohren die M. lim. Hartn. 10/IV à imm.

Fig. 2. Junges Kaninchen. Pacini'sche Flüssigkeit 2 Stunden. Die Riechzellen endigen mit Kolben, welche die Riechhärchen tragen. Hartn. 10/IV à imm.

Fig. 3. Kaninchen; Ameisensäure, Gold-Ameisensäure. Schnitt senkrecht zur Oberfläche. Die Nerven durchbohren die absolut farblose subepitheliale Schicht se und dringen in das Epithel ein. Dr Bowmansche Drüse. Winkel BIIV.

Fig. 4. Flächenschnitt desselben Geruchsorganes. Rechts unten liegt der Schnitt in einer tieferen Schicht, als links. Rechts unten zwischen den verästelten Nervenfasern nur Füsschen von Epithelialzellen ez, links auch die Riechzellenkörper rz erkennbar. Dr Durchschnitte Bowman'scher Drüsen. Winkel 8/IV.

Fig. 5. Junge Ratte, etwa 8 Tage alt; Müller'sche Flüssigkeit 2 Monat; Zupfpräparat. Unter dem Epithel die Schicht der sternförmigen Bindegewebszellen stz. rz, die über die Obertläche herausragenden Riechzellen. Winkel 8/IV. 
Béla Dezsö: Fortsetzung d. Untersuch. üb. Tethya lyncurium Autorum. 151

Fig. 6. Dasselbe Präparat. Eine der sternförmigen Zellen mit 2 Epithelialzellen; deutliche Grenzen zwischen beiden. In dem senkrecht gestrichelten Saum über dem Epithel die peripherischen Riechzellenfortsätze sichtbar. Winkel 8/IV.

Fig. 7. Hausmaus, Kali bichr. 4 pCt. 14 Tage. Die subepithelialen sternförmigen Zellen von der Fläche. Winkel 8/IV.

Fig. 8. Die unter den letzteren gelegene homogene Bindegewcbsschicht mit den Löchern für die Bowman'schen Drüsen. Winkel 8/IV.

\section{Fortsetzung ḋer Untersuchungen über Tethya lyn- curium Autorum.}

Von

Dr. Bela Dezsỏ aus Kolozsvàr.

Hierzu Tafel XII.

Bevor ich die Fortsetzung meiner unter Prof. 0. Schmidt's Leitung ausgeführten Untersuchungen ${ }^{1}$ ) im zoologischen Institute in Graz uber die Tethya lyncurium aus Triest unternahm, habe ich Herrn Prof. F. Eilhard Schulze's Original-Präparate iuber die schon von ihm beschriebenen Spongien studirt und habe sodann, um mich mit seiner Methode bekannt za machen, unter seiner Leitung die von ihm bisher beobachteten Spongien ${ }^{2}$ ) theils an lebenden, theils an conservirten Stiicken nachuntersucht.

1) Dieses Archiv Bd. XVI. 1879. Die Histiologie und Sprossenentwickelung der Tethyen, besonders der Tethya lyncurium, Lieberkühn (Autorum).

2) Sycandra raphanus, H. Aplysina aërophoba, Nardo. Aplysilla sulphurea, F. E. Schulze. Halisarca lobularis, 0. Sch. Halisarca Dujardini, Johnston. Euspongia officinalis adriatica, F. E. Schulze. Cacospongia scalaris, 0. Sch. Spongelia pallescens, 0. Sch. Spongelia avara, 0. Sch. Chondrilla uncula, 0. Sch. Chondrosia reniformis, Nardo. 